\title{
Purificación y caracterización de la ficocianobilina
}

\author{
Purification and characterization of phycocyanobilin
}

Alexey Llopiz ${ }^{1, a}$, Ariadna Milián ${ }^{2, a}$, Gabriela Díaz Domínguez ${ }^{3, b}$

DOI. 10.21931/RB/2016.01.04.8

\section{RESUMEN}

La ficocianobilina es el grupo prostético de las ficobiliproteínas C-ficocianina y Aloficocianina, las que se encuentran presentes en las algas verde-azules. Entre las estrategias empleadas para la purificación de este pigmento tetrapirólico se encuentra la extracción de las proteínas y posterior ruptura del enlace tioéter, mediante el que está unido las cadenas polipeptídicas. Posteriormente se lleva a cabo el proceso de extracción con un solvente orgánico y la purificación basada en la cromatografía en placa, en batch o columna. La caracterización de esta molécula implica métodos cromatográficos, espectroscópicos y la determinación de la actividad antioxidante. En esta revisión presentamos un resumen de las estrategias de purificación empleadas para la obtención de este pigmento, así como los métodos de caracterización fundamentales. Adicionalmente comentamos sobre posibles estrategias que se pudieran emplear para la obtención de preparaciones de la PCB con elevadas purezas, que puedan ser empleadas para la evaluación de sus propiedades potenciales en la industria biofarmacéutica.

Palabras clave: ficocianobilina, tetrapirrol, grupo-prostético, ficobiliproteínas.

\section{ABSTRACT}

Phycocyanobilin is a prosthetic group of phycobiliproteins named C-phycocyanin and allophycocyanin, which are located in blue-green alga. Purification of PCB implies protein extraction, thioeter bond rupture and extraction in organic solvent followed by separation on chromatographic system. Characterization of PCB is accomplished through chromatographic analysis, spectroscopic techniques and antioxidant capacity determinations. In this review, we present different strategies for PCB purification as well as many methods used for pigment characterization. Also, we propose some procedures to obtain this molecule from cellular as starting material to produce PCB in enough quantity to evaluate its biological potential in biopharmaceutical industry.

Keywords: phycocyanobilin, tetrapyrrole, prosthetic group, phycobiliproteins.

\section{Introducción}

La ficocianobilina es el pigmento tetrapirrólico que constituye el grupo prostético de la C-ficocianina (CPC) y la Aloficocianina (APC). Estas proteínas son constituyentes de los ficobilisomas presentes en las algas verde-azules ${ }^{1} \mathrm{y}$ han sido asociadas a propiedades antioxidantes, inmunomoduladoras y citoprotectoras. ${ }^{2-6}$ Adicionalmente se ha demostrado que muchas de estas propiedades biológicas están asociadas al sistema tetrapirrólico que está presente en estas proteínas como grupo prostético. ${ }^{2,7,8}$ Presumiblemente la acción de la PCB está relacionada con la inhibición de la enzima NADPH oxidasa y con su capacidad antioxidante..$^{2,9,10}$ Adicionalmente debido a sus propiedades físico-químicas es posible que esta molécula pueda ejercer su acción traspasando la membrana hemato-encefálica, por lo que potencialmente podría ser un producto con aplicaciones en el tratamiento de la isquemia cerebral. ${ }^{7,11}$

Los métodos descritos para la purificación y caracterización de la PCB implican la extracción previa de las ficobiliproteínas en las que está contenido este grupo prostético. Posteriormente el pigmento es liberado de la estructura polipeptídica mediante el tratamiento con ácido concentrado o reflujo con un alcohol . ${ }^{12,13}$ La extracción posterior se realiza con solventes orgánicos como cloroformo o diclorometano debido a la solubilidad del pigmento en estos sistemas. Finalmente, el proceso de purificación puede implicar un método cromatográfico ya sea en placa, batch o columna. ${ }^{14}$ Una alternativa que emplea herramientas biotecnológicas está asociada a la co-expresión de las enzimas involucradas en la modificación del grupo hemo a Biliverdina (BV) y posteriormente a PCB. ${ }^{9}$ El organismo

\footnotetext{
${ }^{1}$ Licenciado en Bioquímica. Máster en Biotecnología Mención Investigación de nuevos productos.

${ }^{2}$ Técnico en Química Industrial.

${ }^{3}$ Licenciada en Bioquímica y Biología Molecular.

a Departamento de Ciencias Químicas. Carrera de Ingeniería en Biotecnología. Universidad de Las Américas. Quito. Ecuador.

${ }^{\mathrm{b}}$ Instituto de Hematología e Inmunología. La Habana. Cuba.

Correspondencia: Dirección: José Queri, Quito 170137, Ecuador. Correo electrónico: alexey-llopiz@outlook.com y a.llopiz@udlanet.ec.
} 
que se utiliza para la expresión heteróloga de estas enzimas es la bacteria Escherichia coli. La estrategia descrita involucra la obtención del pigmento en su forma libre, por lo que el proceso de purificación comenzaría con la ruptura celular y la extracción con un solvente orgánico del pigmento, así como su posterior separación cromatográfica.

Por otra parte, las técnicas analíticas empleadas para la caracterización de la PCB comprenden métodos cromatográficos, ${ }^{9}$ entre los que se encuentran la RP-HPLC y la TLC. El análisis espectroscópico se lleva a cabo mediante espectroscopía UV-visible (UVvis), fluorescencia, ${ }^{13}$ Infrarojo (IR), ${ }^{15}$ Espectrometría de Masas (MS) $)^{1,9,12}$ y Resonancia Magnética Nuclear (RMN). ${ }^{16}$

Aunque en la literatura científica se han reportado algunas metodologías para la purificación de la ficocianobilina han sido con fines analíticos. De tal forma estas resultan ser trabajosas y difícilmente escalables. También en su mayoría utilizan como materia de partida las ficobiliproteínas (al menos parcialmente purificadas) en lugar de las células de las algas verde-azules donde está contenido este pigmento.

En este trabajo realizamos un análisis sobre los principales métodos descritos para la obtención y caracterización del pigmento ficocianobilina. También se hace énfasis en algunas estrategias que se pudieran desarrollar para su obtención a partir de las células o de preparaciones de ficobiliproteínas con purezas bajas.

\section{Métodos}

Las estructuras químicas obtenidas del Pubchem17 fueron visualizadas y/o analizadas con el programa PC3D (Jandel Scientific, Corte Madera, CA, USA) y las obtenidas del PDB con el programa UCSF Chimera. ${ }^{18}$ Las comparaciones de las secuencias de proteínas se realizaron con las aplicaciones BLAST, SmartBLAST $^{19}$ y CLUSTAL OMEGA. ${ }^{20}$
La ficocianobilina es el grupo prostético de las ficobiliproteínas presentes en Arthrospira platensis

La C-ficocianina y la Aloficocianina son las ficobiliproteínas que forman parte del aparato fotosintético de la cianobacteria Arthrospira platensis. ${ }^{15}$ Estos polipéptidos (CPC/APC) son constituyentes de los ficobilisomas de este microrganismo, y se encuentran aproximadamente en una proporción de 10:1 formando estructuras dodecaméricas y hexaméricas, respectivamente..$^{21,22}$. Ambas ficocianinas tienen unido el cromóforo ficocianobilina (PCB) a cisteínas específicas mediante un enlace tioéter. Lemberg (revisado en el trabajo de $\mathrm{OHEOCHA}^{13}$ ) demostró que la PCB se une de forma covalente a la cadena polipeptídica y que su estructura es semejante a la de un pigmento tetrapirrólico. De hecho, este fue erróneamente identificado como mesoviliverdina. ${ }^{13,23}$

La posición de cada cromóforo fue determinada mediante estudios estructurales ${ }^{24} \mathrm{y}$ el sitio de unión entre el cromóforo y la proteína por Resonancia Magnética Nuclear (RMN). ${ }^{23}$ Para la CPC de A. platensis los sitios de unión son las cisteínas 84 de la cadena alfa, y las 82 y 153 de la cadena beta. La posición de unión del cromóforo en esta proteína es un sitio altamente conservado, de acuerdo al análisis realizado con la aplicación CLUSTAL OMEGA $^{20}$ (Figura 1).

El alineamiento múltiple de secuencia se realizó con los resultados obtenidos del BLAST ${ }^{19}$, que tuvieran al menos un $70 \%$ de identidad con cada una de las estructuras correspondientes a las cadenas de la CPC. Adicional a las posiciones correspondientes de las cisteínas donde se une el grupo prostético PCB, también existen otras regiones de estas proteínas que son altamente conservadas (Figura 2). Presumiblemente esto pudiera estar relacionado con el mantenimiento de la estructura tridimensional que caracteriza a cada una de las cadenas de la CPC.

La comparación de las dos cadenas de la CPC indica un grado de similitud estructural a nivel de estructura primaria de un $28 \%$ (Figura 2A). La diferencia entre ambas cadenas polipeptídicas está dada por inserciones en la cadena beta, en relación a la

\section{Cadena_alfa_CPC \\ Sp|P00308|PHCA1_SYNP6 Sp |Q5N4S9|PHCA2_SYNP6 \\ $\operatorname{tr} \mid$ L8LZG7|L8LZG7_9CYAN \\ $\operatorname{tr} \mid$ K9Z7Q5|K9Z7Q5_CYAAP \\ $\operatorname{tr} \mid$ AOAOD6ADX6|AOAOD6ADX6_9CHRO \\ $\operatorname{tr} \mid$ AOAOD6AUG3|AOAOD6AUG3_9CHRO \\ sp|P20776|PHCA_SYNY1}

\section{Cadena_beta_CPC}

$\operatorname{tr} \mid$ AOAOS3TNS2 |AOAOS3TNS2 9CYAN $\operatorname{tr} \mid$ AOAOD6YLF6 $\mid A 0 A 0 D 6 Y L F 6$ MASLA $\operatorname{tr|AOAOMOSQT8|AOAOMOSQT8\_ 9CYAN}$ $\operatorname{tr}|V 5 V 5 E 1| V 5 V 5 E 1$ 9CHRO $\operatorname{tr} \mid$ Q71RW8|Q71RW8_THEVL Sp |P50033|PHCB_THEEB $\operatorname{tr} \mid$ K9RNE6 $\mid$ K9RWE 6 SYNP3 $\operatorname{tr}|\mathrm{K} 9 \mathrm{SCQ} 3| \mathrm{K} 9 \mathrm{SCQ} 3$ 9CYAN $\operatorname{tr} \mid$ K9VZU9|K9VZU9_9CYAN

$\operatorname{tr} \mid$ AOAOS3TNS2 |AOAOAOS3TNS2_9CYAN
$\operatorname{tr} \mid$ AOAOD6YLF6 |AOAOD6YLF6_MASLA
$\operatorname{tr} \mid$ AOAOMOSQT8|AOAOMOSQT8_9CYAN
$\operatorname{tr} \mid$ V5V5E1 |V5V5E1_9CHRO
$\operatorname{tr} \mid$ Q71RW8 |Q71RW8_THEVL
sp |P50033|PHCB_THEEB
$\operatorname{tr} \mid$ K9RWE6 |K9RWE6_SYNP3
$\operatorname{tr} \mid$ K9SCQ3 |K9SCQ3_9CYAN
$\operatorname{tr} \mid$ K9VZU9|K9VZU9_9CYAN

YSKFPYTTSTPGNNFASTPEGKAKCARDIGYYRIVTYALVAGGTGPIDEYLLAGLDEIN YSKFPYTTSTPGNNFASTPEGKAKCARDIGYLRIVTYALVAGGTGPIDEYLLAGLDEIN YSKFPYTNSTPGPSYASTPEGKAKCSRDIGYYLRMVTYCLISGGTGPMDEYLIAGLDEIN YNKFPYTTSTPGSOYASTAEGKAKCARDIGYYLRMVTYCCVAGGTGPMDEYLIAGIDEIN YSKFPYTTTTPGANFASTAEGKAKCSRDIGYYRMVTYCLVAGGTGPMDEYLIAGIDEIN YSKFPYTTTTPGANFASTAOGKAKCSRDIGYYLRMVTYCLVAGGTGPIDEYLIAGIDEIN YSKFPYTTTTPGANFASTAOGKAKCSRDIGYYLRMVTYCLVAGGTGPIDEYLIAGIDEIN $*^{*}:^{*}:^{*} \quad:{ }^{* *} * * * *::^{*}:^{*}:{ }^{*}:$. $^{* *}:^{*}:{ }^{*}:{ }^{*}::^{*}$ $:: \quad * * *$

FEEQPOLISPGGNAYTNRRMAACLRDMEIILRYTYAILAGDASVLDDRCLNGLRETYOA FEEQPOLISPGGNAYTNRRMSACLRDMEI ILRYTTYAILAGDASVLDDRCLNGLRETYQA FEEQPQLISPGGNAYTNRRIISACLRDMEIILRWTYAILAGDASVLDDRCLNGLRETYQA FAEQPQLIQPGGNAYTNRRMAACLRDMEIILRYTYAILAGDSSVLDDRCLNGLRETYQA FAEQPQLIQPGGNAYTNRRMAACLRDMEI ILRYTYYALAGDSSVLDDRCLNGLRETYQA FAEQPQLIQPGGNAYTNRRMAACLRDMEIILRYTYAILAGDSSVLDDRCLNGLRETYQA FAEQPOLIQPGGNAYTNRRMAACLRDMEIILRYTYYATLAGDSSVLDDRCLNGLRETYQA FEEQPQLIQPGGNAYTSRRMAACLRDMEIILRYTYATMAGDSSVLDDRCLNGLRETYQA FEDQPQLIQPGGNAYTNRRIMACLRDMEIILRWTYATIAGDSSVLDDRCLNGLRETYQA : $: * * * * * * * *: * *::^{*}: * * *::^{*}: \quad * *:: * *, * * * * ; * * *$$$
\uparrow
$$

LGTPGSSVAVGVÖKMKEAAINIANDPNGITKGDCSQ̈LMSEVASYFDRAAAAVA LGTPGSSVAVGVQKMKEAAVGIANDPNGITKGDCSALISEVASYFDRAAAAVA LGTPGSSVAVGVQKMKEAAVGIANDPNGITKGDCSALMSEVAGYFDRAAAAVA LGTPGSSVAVAIQKMKDAAIAIANDPNGITPGDCSALMSEIAGYFDRAAAAVA LGTPGSSVAVAIQKMKDAAIAIANDPNGITPGDCSALMSEIAGYFDRAAAAVA LGTPGSSVAVAIQKMKDAAIAIANDPNGITPGDCSALMSEIAGYFDRAAAAVA LGVPGSSVATGVSKMIKDAAIAVANDPNGIVPGDCSSLISEVAGYFDRAAAAVA LGVPGGSVAAGVQKMKEAAIAVANDSAGITPGDCSALMSELAGYFDRAASAVA LGVPGGSVASGVOKMKDAAIAIANDPNGITKGDCSALMSELSGYFDRAASAVA

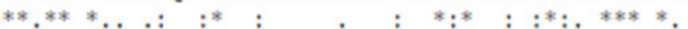

Fig. 1. Fragmentos de los resultados obtenidos del análisis de "alineamiento múltiple de secuencia" con el CLUSTAL OMEGA de varias secuencias aminoacídicas de la C-ficocianina. Las cisteínas donde está presente el enlace tioéter se señalan con flechas. 
estructura de la cadena alfa. Estas inserciones implican dos regiones específicas: 73-74 y 146-155. Este cambio de la secuencia en las dos cadenas presumiblemente está asociado a la acomodación de otro cromóforo en la cadena beta. ${ }^{21}$
La estructura está conformada por dos grupos carboxilos y adicionalmente contiene varias insaturaciones conjugadas, las que probablemente están relacionadas con sus propiedades antioxidantes. ${ }^{26}$ También están presentes ls grupos aminas en los

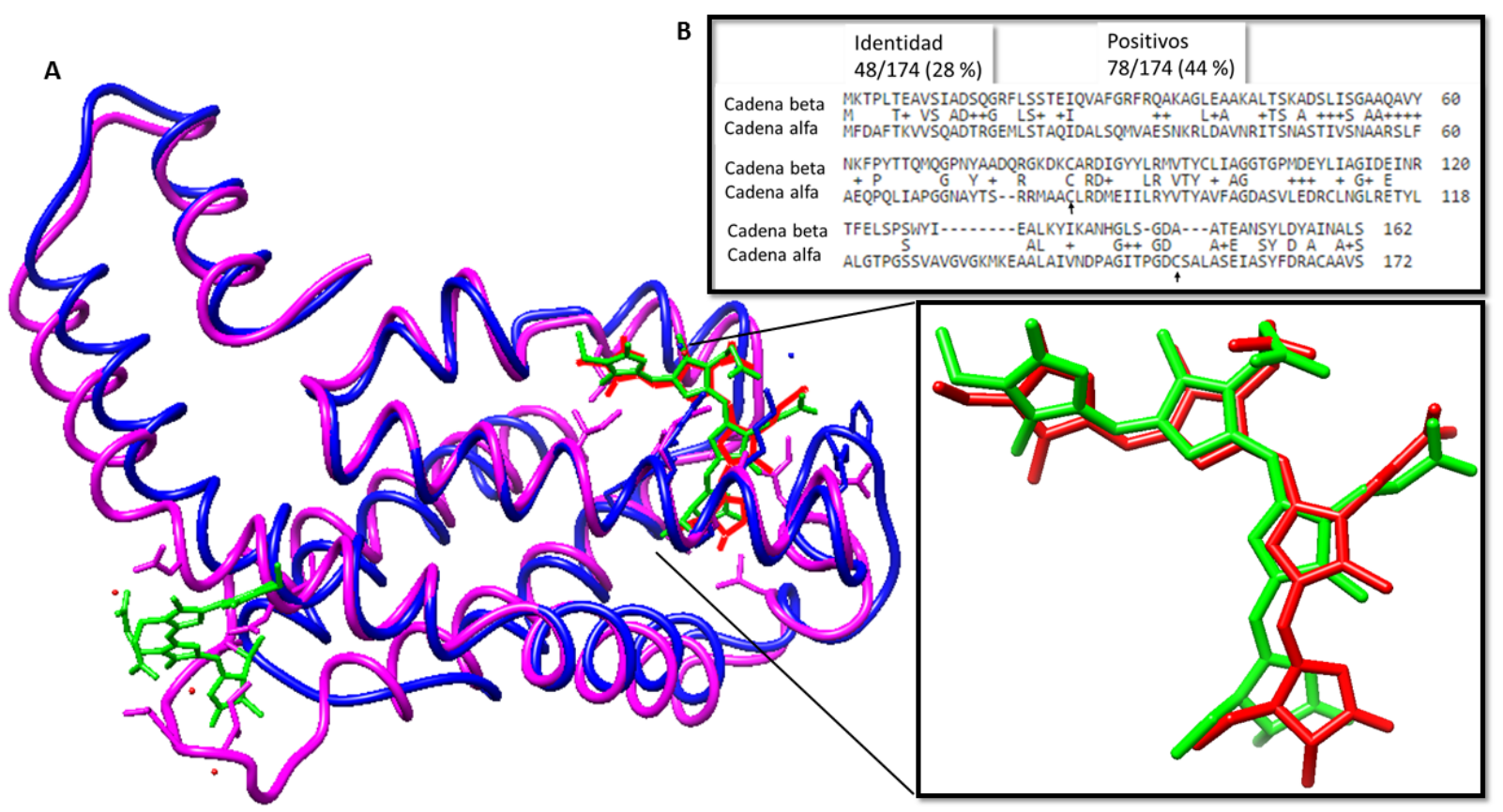

Fig. 2. Comparación de las estructuras de las cadenas alfa y beta de la CPC de A. platensis. A) Alineamiento de las secuencias de las cadenas alfa y beta de la CPC. El cromóforo se une en las posiciones 84 de la cadena alfa, y en las 82 y 153 de la cadena beta. B) Superposición de las estructuras tridimensionales de las cadenas alfa (azul) y beta (magenta) de la CPC. En la ampliación de la superposición de la estructura de los cromóforos de la cadena alfa (mostrado en rojo) y de la cadena beta (mostrado en verde) se observa que ocupan básicamente la misma posición espacial. Las estructuras fueron obtenidas del Protein Data Bank ${ }^{25}$ (1GHO).

Las dos cadenas de la CPC también son conservadas en relación a sus estructuras tridimensionales (Figura 2B). Incluso en el contexto del posicionamiento del grupo prostético en la cadena polipeptídica existe una elevada complementariedad (ampliación en Figura 2B). ${ }^{21}$

\section{Estructura de la ficocianobilina}

La ficocianobilina corresponde a la de un sistema conjugado formado por cuatro anillos pirrólicos, denotadas por las letras A-D. Para esta estructura pueden existir dos estereoisómeros ( $\mathrm{Z}$ y E) en la posición 3 (Figura 3, PubChem CID 6438349).

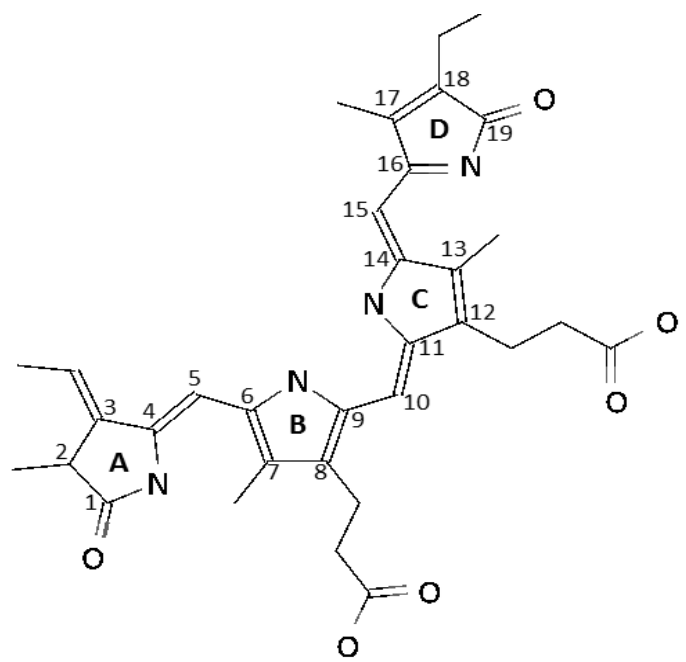

Fig. 3. Estructura de la ficocianobilina, el grupo prostético de las ficobiliproteínas C-ficocianina y aloficocianina. El sistema tetrapirrólico está conformado por cuatro anillos ( $B, C$ y $D$ ) que dan lugar a un compuesto con inaturaciones conjugadas. anillos centrales (B y C) y amidas en los ubicados en los extremos (A y D). La unión del cromóforo y cada cisteína de las cadenas polipeptídicas ocurre en el C3' del anillo A. ${ }^{27,28}$

\section{Purificación de la ficocianobilina}

El proceso de purificación de la ficocianobilina depende del material de partida del que se extraerá este pigmento. Sin embargo, este se obtiene mayoritariamente de preparaciones de ficobiliproteínas con diferentes grados de pureza. ${ }^{1,13,29,30}$ Cuando la PCB es obtenida a partir de la proteína al menos parcialmente purificada, se eliminan sustancias orgánicas poco solubles en el medio acuoso empleado para la extracción de las proteínas. Una alternativa a este material de partida sería obtener el pigmento directamente a partir de las células en las que se encuentra contenido la PCB, lo cual está poco descrito en la literatura científica. En este caso también se realizaría el proceso de extracción de las proteínas en un medio acuoso. Posteriormente las proteínas se pudieran precipitar con sulfato de amonio ${ }^{31}$ o medio ácido y lavar el material insoluble con un solvente orgánico, previo al proceso de liberación del pigmento de la cadena polipeptídica, el que implica calentamiento a temperaturas sobre los $40{ }^{\circ} \mathrm{C} .{ }^{14}$ Contrariamente si el proceso se llevara a cabo con un solvente orgánico (como un alcohol) y calentamiento, este disgrega la estructura de la membrana citoplasmática y extrae todos los compuestos que sean solubles en este medio.

\section{Ruptura del enlace tioéter}

La ficocianobilina fue obtenida por primera vez, por Lemberg en 1930, a partir de la liberación de la cadena polipeptídica de la CPC mediante calentamiento de esta proteína con ácido clorhídrico concentrado a $80^{\circ} \mathrm{C}$ en ausencia de aire. Aunque este constituye un método establecido para la obtención de este pigmento, se debe tener en cuenta que el tratamiento con ácido con- 
centrado produce diferentes estructuras, en función del tiempo de tratamiento y la concentración del ácido. ${ }^{13}$ Otro procedimiento empleado para la obtención de $\mathrm{PCB}$ es el reflujo de $\mathrm{HBr}$ en una solución de CPC/ TFA1.

En 1962 Fujita y Hattori informaron que este tetrapirrol puede ser obtenido por reflujo de la ficobiliproteína con metanol neutro. ${ }^{23} \mathrm{La}$ reacción de metanólisis puede ser optimizada al realizarla a $40^{\circ} \mathrm{C}$ en la oscuridad y en presencia de cloruro mercúrico. Sin embargo, se debe tener en cuenta que las sales mercúricas son sustancias con toxicidad comprobada. Esta reacción de alcohólisis también puede ocurrir con otros solventes como el etanol o el 1-butanol. ${ }^{32}$ Debido a que las ficobiliproteínas en las que se encuentran la $\mathrm{PCB}$ como grupos prostéticos son poco solubles en alcoholes y a la naturaleza del enlace tioéter, las reacciones de alcohólisis se deben llevar a cabo a temperaturas cercanas al punto de ebullición del alcohol, con reflujo y/o agitación. ${ }^{14}$

Adicionalmente existen estrategias para la obtención del grupo prostético en el contexto de una estructura peptídica. Estas implican el tratamiento de las proteínas con enzimas proteolíticas como subtitlisina, ${ }^{14}$ pepsina ${ }^{33} y$ tripsina. ${ }^{23,34,35}$

\section{Obtención mediante métodos biotecnológicos}

La PCB también ha sido obtenida en E. coli por la co-expresión de la hemo-oxigenasa. Esta enzima está relacionada con la formación del pigmento BV a partir del grupo hemo. Posteriormente este pigmento es reducido para dar lugar a la $\mathrm{PCB}$, proceso catalizado por la enzima ficocianobilina:ferredoxina oxidoreductasa. ${ }^{36-38}$ Los resultados de Ge et al. están relacionados específicamente con la obtención de la PCB libre por vía recombinante. ${ }^{9}$ Luego de la obtención del pigmento en E. coli, este es extraído con metanol y posteriormente purificado mediante RP-HPLC. La pureza obtenida fue de un $95 \%$ (de acuerdo al análisis mediante RP-HPLC en condiciones analíticas) y un rendimiento final aproximado de $0.3 \mathrm{mg}$ de $\mathrm{PCB} / \mathrm{L}$ de cultivo. Aunque el objetivo de esta revisión está relacionado con la obtención de la PCB, cabe mencionar que también existen estrategias que implican la formación de las estructuras proteicas que contienen el pigmento unido de modo covalente. ${ }^{39}$

\section{Extracción de la ficocianobilina con solventes orgá- nicos}

Aunque las ficobiliproteínas son estructuras hidrosolubles, la solubilidad en agua del pigmento liberado luego de la ruptura del enlace tioéter es relativamente baja. Sin embargo, la solubilización de la PCB en solventes orgánicos es posible, ${ }^{9}$ en especial si el diácido obtenido se esterifica con un alcohol como el metanol. ${ }^{14}$ Entre las fases orgánicas más empleadas para la extracción del pigmento se encuentran el diclorometano, 1-butanol y cloroformo ${ }^{1.9,13}$ Los sistemas más empleados para la obtención de las dos fases (orgánica y acuosa) implica el uso de embudos separadores para la recuperación de la fase orgánica componente de la molécula de interés. Por otro lado, si se desea acelerar el proceso de formación de las dos fases se pudiera realizar mediante centrifugación.

\section{Purificación mediante métodos cromatográficos}

La ficocianobilina y sus derivados pueden ser separados mediante cromatografía en gel de silica con una fase móvil isocrática conformada por una mezcla de solventes como benceno/acetato de etilo/agua en una proporción 80/20/0.13 (v/v). ${ }^{14}$ La separación implica dos isómeros del éster metílico de la PCB, así como el aducto formado con el metanol. Estas separaciones se pueden realizar en dos modos: (1) cromatografía en placa delgada (TLC, del inglés Thin Layer Chromatography) o (2) mediante cromatografía líquida (LC, del inglés Liquid Chromatography) en una columna pre-empacada o en batch. Adicionalmente se pudieran emplear otros sistemas que implican cromatografía líquido-líquido (CC, del inglés Countercurrent Chromatography). ${ }^{40}$

Otra de las técnicas empleadas para la purificación de la fi- cocianobilina implica el uso de la cromatografía en fase reversa (RP). En este sistema generalmente se emplean columnas de C18 y las fases móviles pueden estar conformadas por solventes orgánicos como el metanol, acetonitrilo, ${ }^{9}$ acetona, ${ }^{41}$ etanol/ácido acético. ${ }^{16}$

Estrategias generales para la purificación de la ficocianobilina a partir de células de A. platensis

La mayoría de los procedimientos descritos para la purificación de ficocianobilina a partir de fuentes naturales no permiten obtener grandes cantidades de este pigmento. Este hecho está relacionado con dos elementos fundamentales: (1) la proporción de la PCB en el sistema natural del que se extrae y (2) difícil escalabilidad de los procedimientos establecidos.

El proceso de purificación de la ficocianobilina tiene una serie de etapas (Figura 4) que comienzan con la extracción del sistema celular en el que está contenido este pigmento. La mayoría de las estrategias implican la purificación de las ficobiliproteínas como un paso previo a la liberación del pigmento de la fracción polipeptídica. Posteriormente se emplean varios pasos de extracción en dos fases inmiscibles y/o procesos cromatográficos. Sin embargo, estos procedimientos se han realizado en su mayoría con la finalidad de estudios estructurales y/o funcionales de la ficocianobilina.

En la literatura científica están escasamente descritas las estrategias adecuadas para la obtención de este pigmento a partir de células de cianobacterias o algas. Una alternativa muy conveniente para la purificación de la PCB implicaría la ruptura celular en un medio acuoso y ácido (TFA, TCA, $\mathrm{HCl}$, etc.), en el que se extraerían algunas proteínas (y otras podrían precipitar). De esta forma, a la vez se propiciaría la ruptura del enlace tioéter, y la liberación correspondiente de la PCB (Figura 4). Alternativamente se pudieran romper las células en un medio acuoso y precipitar las proteínas extraídas en un medio salino o ácido. Posteriormente se lavarían con metanol para eliminar contaminantes de naturaleza orgánica, como la clorofila y otros pigmentos que se solubilizan a temperatura ambiente. Sólo al calentar a temperaturas sobre los $40{ }^{\circ} \mathrm{C}$ se favorecería el proceso de ruptura del enlace tioéter y la liberación del pigmento de interés. ${ }^{14} \mathrm{Si}$ el proceso de obtención del pigmento se lleva a cabo por vía biotecnológica entonces no se tendría que llevar a cabo el proceso de metanólisis, ya que esta molécula se obtendría en un modo no conjugado. ${ }^{9}$

Una vez ocurrido el proceso de metanólisis los componentes proteicos se obtendrían como un precipitado luego de centrifugar. En el material soluble estaría presente la PCB, junto a contaminantes que deben ser eliminados mediante extracción en sistemas líquidos bifásicos. Otro elemento asociado a este proceso debe ser un paso cromatográfico. Entre las alternativas más adecuadas se encuentran las cromatografías líquidas ya sean en fase normal (gel de silica) ${ }^{14} \mathrm{o}$ en fase reversa (RP). ${ }^{9}$ También pudieran realizarse procesos cromatográficos que impliquen partición entre dos fases líquidas (estacionaria y móvil), así como separaciones en placas delgadas (TLC). ${ }^{42}$

\section{Caracterización de la ficocianobilina Métodos cromatográficos}

La cromatografía en fase reversa (RP-HPLC) es el método cromatográfico fundamental para la caracterización de las preparaciones de PCB. ${ }^{12,16,41}$ Esta técnica permite la separación de productos con elevada similitud estructural con la $\mathrm{PCB}$, entre los que se encuentran la BV y el aducto PCB-metanol. Otras de las alternativas cromatográficas que pueden tener aplicaciones analíticas es la configuración en placa delgada (TLC). ${ }^{42}$

\section{Métodos espectroscópicos}

Debido a la presencia del sistema de enlaces múltiples conjugados, este pigmento presenta un máximo absoluto de absorción alrededor de los $360 \mathrm{~nm}$ y un máximo relativo cerca de los 


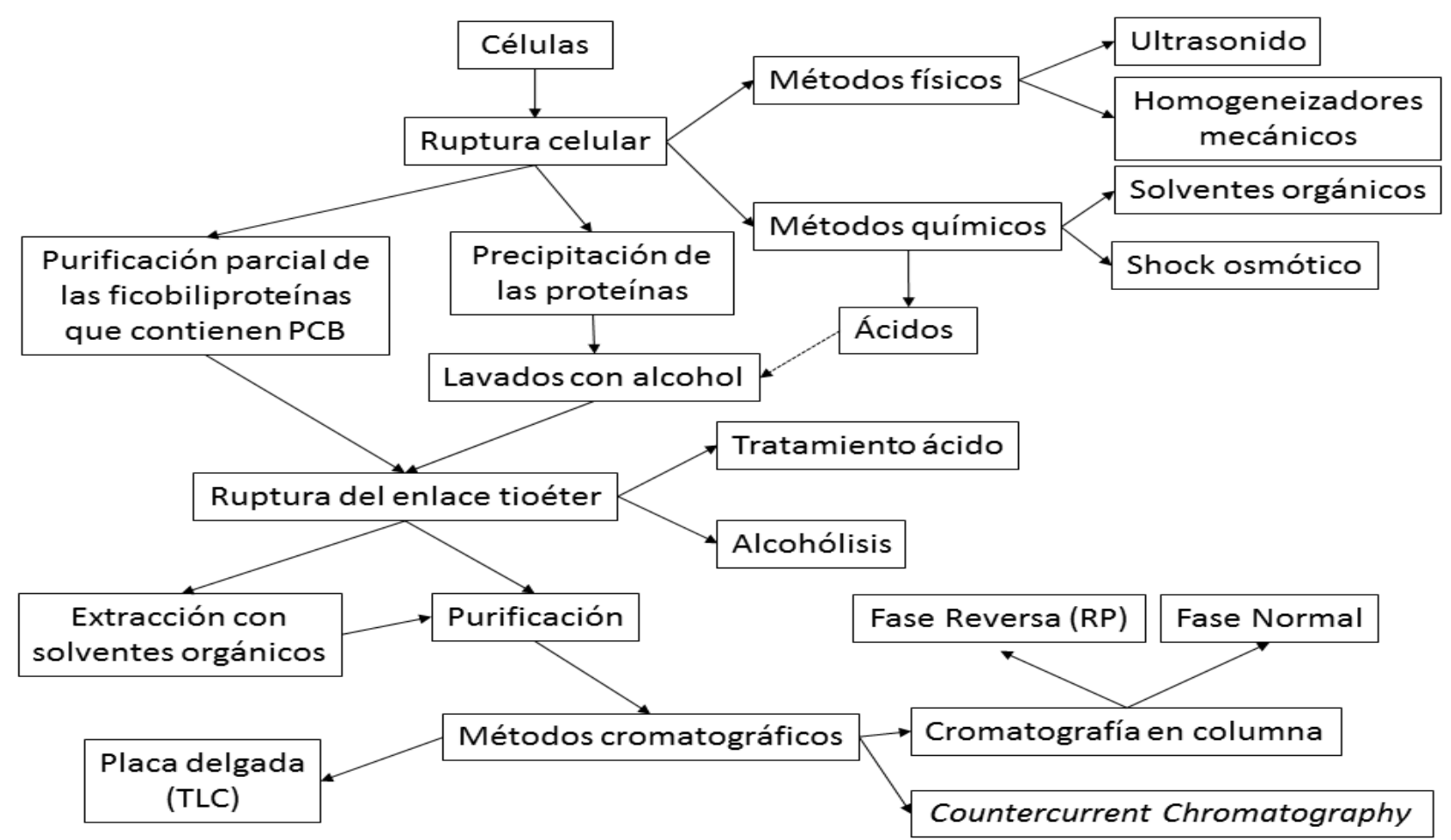

Fig. 4. Estrategia general para la extracción y purificación de la ficocianobilina a partir de células de cianobacterias y algas.

$620 \mathrm{~nm}$. Sin embargo, se debe mencionar que el micro-entorno en el que se encuentra el pigmento determina el máximo de absorción absoluto. ${ }^{1}$ Cuando está formando parte de la CPC este valor se encuentra alrededor de los $620 \mathrm{~nm}$, pero si el sitio donde se encuentra unida es en la APC el máximo de absorbancia tiene un corrimiento hasta los $650 \mathrm{~nm}$. El espectro de fluorescencia de la PCB muestra un máximo de emisión alrededor de los $678 \mathrm{~nm}$, el cual se desplaza hasta un valor de $633 \mathrm{~nm}$ en presencia de cinc. ${ }^{13}$

El espectro IR de la ficocianobilina presentó un máximo en $3470 \mathrm{~cm}^{-1}$, lo que indica la presencia de grupos hidroxilos en la estructura. La señal a $1462 \mathrm{~cm}^{-1}$ es indicativa del grupo carbonilo, el cual se encuentra en forma de ácidos carboxílicos y amidas. La señal a $1461 \mathrm{~cm}^{-1}$ está asociada al grupo isoprolilo y la de $850 \mathrm{~cm}^{-1}$ a enlaces $\mathrm{C}-\mathrm{H}$ de sistemas aromáticos o heterociclos. ${ }^{15}$ También se detectaron otras señales a 1157, 1116, 1032, 602 y $190 \mathrm{~nm}$, las que no fueron asignadas.

La estructura química de la PCB fue establecida mediante espectrometría de masas (MS). En este estudio la PCB fue obtenida mediante reflujo con metanol y tratamiento con ácido concentrado. Luego el producto fue extraído en cloroformo y esterificado. El éster volátil fue separado en gel de silica y analizado por $\mathrm{MS}^{1.14}$ La relación $\mathrm{m} / \mathrm{z}$, determinada mediante $\mathrm{FAB}$ (del inglés Fast Atom Bombardment) fue de 586 Th y 588 Th para la PCB sin esterificar. ${ }^{15}$ Cuando se emplea un método de ionización "suave" (MALDI-TOF) la $\mathrm{m} / \mathrm{z}$ es $587.2 \mathrm{Th}$, estructura que corresponde también al análisis de la preparación de PCB sin esterificar. ${ }^{9}$

\section{Actividad antioxidante}

La actividad antioxidante de la PCB ha sido determinada por los métodos basados en el DPPH ${ }^{9}$ y en el ORAC. ${ }^{2}$ También se ha evaluado el efecto de esta molécula en la protección de lípidos ante la oxidación por AAPH ${ }^{10.29}$

En conclusión, la ficocianobilina es un sistema tetrapirrólico presente en las ficobiliproteínas aloficocianina y C-ficocianina, que tiene aplicaciones potenciales en la industria biofarmacéututica por sus propiedades inmunomoduladoras, citoprotectoras y antioxidantes. Entre las materias primas empleadas para obtener este pigmento se encuentran las cianobacterias y las algas en las que se encuentran las ficbiliproteínas denominadas ficocianinas. Adicionalmente, la ficocianobilina puede ser obtenida también por métodos biotecnológicos, donde la bacteria $E$. coli ha sido uno de los sistemas más empleados. Por otra parte, aunque existen varias estrategias que implican la obtención de PCB con purezas elevadas, la mayoría de estos tienen el inconveniente de ser poco escalables, además de que los rendimientos son bajos. En este trabajo se proponen estrategias que implicarían la obtención de PCB a partir de un sistema celular, el cual pudiera constituir un proceso escalable.

\section{References}

1. Schram, B. L. \& Kroes, H. H. Structure of Phycocyanobilin. Eur. J. Biochem. 19, 581-594 (1971).

2. Benedetti, S., Benvenuti, F., Scoglio, S. \& Canestrari, F. Oxygen radical absorbance capacity of phycocyanin and phycocyanobilin from the food supplement Aphanizomenon flos-aquae. J. Med. Food 13, 223-7 (2010).

3. Bhat, V. B. \& Madyastha, K. M. C-phycocyanin: a potent peroxyl radical scavenger in vivo and in vitro. Biochem. Biophys. Res. Commun. 275, 20-5 (2000)

4. Marín-Prida, J. et al. C-Phycocyanin protects SH-SY5Y cells from oxidative injury, rat retina from transient ischemia and rat brain mitochondria from $\mathrm{Ca} 2+/$ phosphate-induced impairment. Brain Res. Bull. 89, 159-167 (2012).

5. Pentón-Rol, G. et al. C-Phycocyanin ameliorates experimental autoimmune encephalomyelitis and induces regulatory T cells. Int. Immunopharmacol. 11, 29-38 (2011).

6. Pentón-Rol, G. et al. C-Phycocyanin is neuroprotective against global cerebral ischemia/reperfusion injury in gerbils. Brain Res. Bull. 86, 42-52 (2011).

7. Marín-Prida, J. et al. Phycocyanobilin promotes PC12 cell survival and modulates immune and inflammatory genes and oxidative stress markers in acute cerebral hypoperfusion in rats. Toxicol. Appl. Pharmacol. 272, 49-60 (2013)

8. Mccarty, M. F. \& Longevity, C. Phycocyanobilin from Spirulina - the Master Antioxidant. 3-8

9. Ge, B. et al. Combinational biosynthesis of phycocyanobilin using genetically-engineered Escherichia coli. Biotechnol. Lett. 35, 689693 (2013).

10. Tanaka, M. Radical Scavenging Activity of Phycocyanobilin Spirulina Prepared from the Cyanobacterium, Takashi platensis Niizeki, and Morihiko Spirulina platensis contains phycocyanin as a light-har vesting protein for photosynthesis. Phycocyanin has an in tense b. 65, 971-972 (1999).

11. McCarty, M. F., Barroso-Aranda, J. \& Contreras, F. Oral phycocyanobilin may diminish the pathogenicity of activated brain microglia 
in neurodegenerative disorders. Med. Hypotheses 74, 601-5 (2010). 12. Fu, E., Friedman, L. \& Siegelman, H. W. Mass-spectral identification and purification of phycoerythrobilin and phycocyanobilin. Biochem. J. 179, 1-6 (1979).

13. OHEOCHA, C. SPECTRAL PROPERTIES OF THE PHYCOBILINS. I. PHYCOCYANOBILIN. Biochemistry 3, 1343-50 (1964).

14. Beuhler, R. J., Pierce, R. C., Friedman, L. \& Siegelman, H. W. Cleavage of phycocyanobilin from C-phycocyanin. Separation and mass spectral identification of the products. J. Biol. Chem. 251, 2405-11 (1976).

15. Aboshady, A. M. Spectroscopic Study of Phycocyanobilin from the Cyanobacterium Spirulina-Platensis. Biol. Plant. 37, 631-633 (1995).

16. Beale, S. I. \& Cornejo, J. Biosynthesis of Phycobilins. J Biol Chem 266, 22328-22332 (1991)

17. No Title. at <https://pubchem.ncbi.nlm.nih.gov/>

18. Pettersen, E. F. et al. UCSF Chimera--a visualization system for exploratory research and analysis. J. Comput. Chem. 25, 1605-12 (2004).

19. No Title. at <http://blast.ncbi.nlm.nih.gov/Blast.cgi?PROGRAM=bl>

20. No Title. at $<$ http://www.ebi.ac.uk/Tools/msa/clustalo/>

21. Padyana, A. K., Bhat, V. B., Madyastha, K. M., Rajashankar, K. R. \& Ramakumar, S. Crystal structure of a light-harvesting protein C-phycocyanin from Spirulina platensis. Biochem. Biophys. Res. Commun. 282, 893-8 (2001).

22. MacColl, R., Lee, J. J. \& Berns, D. S. Protein aggregation in C-phycocyanin. Studies at very low concentrations with the photoelectric scanner of the ultracentrifuge. Biochem. J. 122, 421-6 (1971).

23. Arciero, D. M., Dallas, J. L. \& Glazer, A. N. In vitro attachment of bilins to apophycocyanin. II. Determination of the structures of tryptic bilin peptides derived from the phycocyanobilin adduct. J. Biol. Chem. 263, 18350-7 (1988).

24. Williams, V. P. \& Glazer, A. N. Structural studies on phycobiliproteins. I. Bilin-containing peptides of C-phycocyanin. J. Biol. Chem. 253, 202-11 (1978).

25. No Title. at <http://www.rcsb.org/pdb/explore/explore.do?structureId=1GH0>

26. van den Berg, J. J. M., Cook, N. E. \& Tribble, D. L. Reinvestigation of the antioxidant properties of conjugated linoleic acid. Lipids 30, 599-605 (1995).

27. van Thor, J. J., Mackeen, M., Kuprov, I., Dwek, R. A. \& Wormald, M. R. Chromophore Structure in the Photocycle of the Cyanobacterial Phytochrome Cph1. Biophys. J. 91, 1811-1822 (2006).

28. Bishop, J. E. et al. Phycobiliprotein-bilin linkage diversity. I. Structural studies on A- and D-ring-linked phycocyanobilins. J. Biol. Chem. 261, 6790-6 (1986).

29. Hirata, T., Tanaka, M., Ooike, M., Tsunomura, T. \& Sakaguchi, M. Antioxidant activities of phycocyanobilin prepared from Spirulina platensis. J. Appl. Phycol. 12, 435-439 (2000).
30. Chapman, D. J., Cole, W. J. \& Siegelman, H. W. Cleavage of Phycocyanobilin From C-Phycocyanin. Biochim. Biophys. Acta 153, 692-698 (1968).

31. Wang, H. The C-Phycocyanin / Beta Protein Inhibits Cancer Cell Proliferation. (2008).

32. Fu, E., Friedman, L. \& Siegelman, H. W. Mass-spectral identification and purification of phycoerythrobilin and phycocyanobilin. Biochem. J. 179, 1-6 (1979).

33. Minic, S. L. et al. SC. J. Proteomics (2016). doi:10.1016/j. jprot.2016.03.043

34. Wallin, R., Selset, R. \& Sletten, K. Characterization of chromophoric peptides from C-phycocyanin. Biochem. Biophys. Res. Commun. 81, 1319-28 (1978).

35. Bishop, J. E. et al. Phycobiliprotein-Bilin Linkage Diversity. J. Biol. Chem. 261, 6790-6796 (1986).

36. Mukougawa, K., Kanamoto, H., Kobayashi, T., Yokota, A. \& Kohchi, T. Metabolic engineering to produce phytochromes with phytochromobilin, phycocyanobilin, or phycoerythrobilin chromophore in Escherichia coli. FEBS Lett. 580, 1333-1338 (2006).

37. Gambetta, G. A. \& Lagarias, J. C. Genetic engineering of phytochrome biosynthesis in bacteria. Proc. Natl. Acad. Sci. 98, 1056610571 (2001).

38. Beale, S. I. \& Cornejo, J. Enzymic Transformation of Biliverdin to Phycocyanobilin by Extracts of the Unicellular Red Alga Cyanidium caldarium. Plant Physiol. 76, 7-15 (1984).

39. Guan, X. et al. Combinational Biosynthesis of a Fluorescent Cyanobacterial Holo- $\alpha$-Phycocyanin in Escherichia coli by Using One Expression Vector. Appl. Biochem. Biotechnol. 142, 52-59 (2007).

40. Du, Q., Jerz, G. \& Winterhalter, P. Isolation of two anthocyanin sambubiosides from bilberry (Vaccinium myrtillus) by high-speed counter-current chromatography. J. Chromatogr. A 1045, 59-63 (2004).

41. Beale, S. I. \& Cornejo, J. Biosynthesis of phycobilins. Ferredoxin-mediated reduction of bilverdin catalyzed by extracts of Cyanidium caldarium. J. Biol. Chem. 266, 22328-22332 (1991).

42. Bishop, J. E., Nagy, J., Connell, J. F. O. \& Rapport, H. Diastereoselective Synthesis of Phycocyanobilin-Cysteine Adducts. J. Am. Chem. Soc. 113, 8024-8035 (1991).

Recibido: 15 de marzo de 2016. Aprobado: 21 de mayo de 2016 